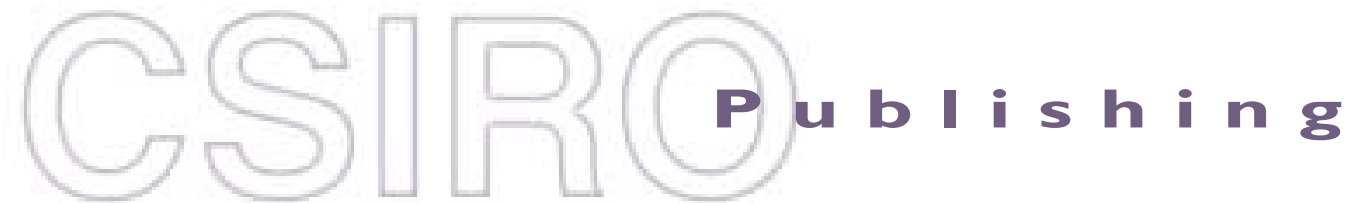

\section{Publications of the Astronomical Society of Australia}

Volume 19, 2002

(C) Astronomical Society of Australia 2002

An international journal of astronomy and astrophysics

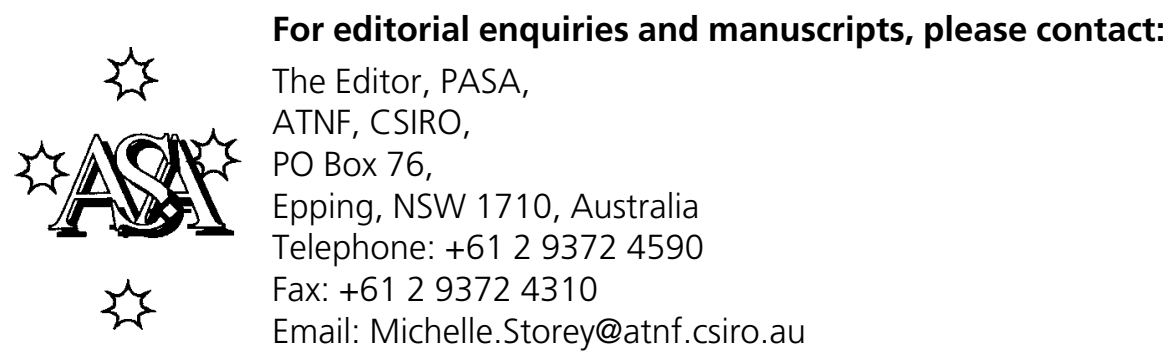

For general enquiries and subscriptions, please contact: CSIRO Publishing PO Box 1139 (150 Oxford St)

Collingwood, Vic. 3066, Australia

Telephone: +6139662 7666

Fax: +61 396627555

Email: publishing.pasa@csiro.au

C S I RO

PUBLISHING Published by CSIRO Publishing

for the Astronomical Society of Australia

www.publish.csiro.au/journals/pasa 


\title{
Millimetre Science with the Upgraded Australia Telescope
}

\author{
T. Wong ${ }^{1}$ and A. Melatos ${ }^{2}$ \\ ${ }^{1}$ CSIRO Australia Telescope National Facility, PO Box 76, Epping NSW 1710, Australia \\ Tony.Wong@csiro.au \\ ${ }^{2}$ School of Physics, University of Melbourne, Parkville Vic 3010, Australia \\ a.melatos@physics.unimelb.edu.au
}

Received 2002 April 26, accepted 2002 July 14

\begin{abstract}
A new astronomical window into the southern skies has been opened with the high-frequency upgrade to the Australia Telescope Compact Array (ATCA), which allows radio-interferometric mapping of sources at wavelengths as short as $3 \mathrm{~mm}$. In anticipation of the upgrade's completion, a two-day workshop was held at the University of Melbourne in November 2001. The workshop covered a diverse range of fields, tied together by a common theme of identifying key areas where ATCA observations can have an impact. More than half of the talks were concerned with molecular clouds and star formation, with the remainder covering topics such as molecular gas in the Galactic Centre, Seyfert nuclei, and high-redshift objects. Some early results from the 3 and $12 \mathrm{~mm}$ prototype systems were also presented. In consultation with the speakers, we are presenting in this article a summary of the talks. The original slides are available from the ATNF website.
\end{abstract}

Keywords: instrumentation: interferometers — ISM: molecules — stars: formation — galaxies: ISM — galaxies: high-redshift — masers

\section{Introduction}

Millimetre ( $\mathrm{mm}$ ) astronomy has grown rapidly in the past decade, spurred by advances in receiver sensitivity and growing scientific interest in what might be termed the 'cold universe'. While molecular lines are still the 'bread and butter' of mm astronomy, a wide range of continuum sources can also be studied at these wavelengths, and today $\mathrm{mm}$ astronomy spans nearly the full range of astrophysical research, from solar system studies to cosmology.

The success of cm-wave interferometry, demonstrated by facilities such as the Westerbork Synthesis Radio Telescope (WSRT), led to the development of mm interferometers in the 1970s and 1980s. There are currently four dedicated $\mathrm{mm}$ arrays, all located in the northern hemisphere at latitudes of $35-45^{\circ}$. A much larger array, to be built in Chile by an international consortium, will provide a manifold increase in collecting area over all existing mm arrays put together. This Atacama Large Millimeter Array (ALMA) will be completed around 2011. In the meantime, however, another $\mathrm{mm}$ array is making its debut in the southern hemisphere: the Australia Telescope Compact Array (ATCA), being upgraded to operate at wavelengths as short as $3 \mathrm{~mm}$, is poised to open a new astronomical window into the southern skies.

In anticipation of the completion of the ATCA upgrade, a workshop on mm-wave science was convened at the University of Melbourne on 29-30 November 2001. Participants from the Australian astronomical community, as well as guests from overseas, came to discuss and learn about areas of science that could be addressed with the new ATCA. As is inevitable with a small meeting, many areas could not be properly represented including, but not limited to, cosmology and VLBI - so this summary should be considered only a sampling of what might be possible. We also apologise in advance if, in trying to summarise the meeting in a cohesive way, we have been rather selective in our recollections. Slides from the talks are available for download from the ATNF website (http://www.atnf.csiro.au/whats_on/workshops/ mm_science2001).

\section{The ATCA as a Millimetre Interferometer}

The ATCA, situated near Narrabri, New South Wales (longitude $150^{\circ} \mathrm{E}$, latitude $30^{\circ} \mathrm{S}$ ), consists of six $22 \mathrm{~m}$ diameter Cassegrain antennas. Five of the antennas are transportable along a $3 \mathrm{~km}$ long east-west track - now supplemented by a $200 \mathrm{~m}$ north spur extending from the centre of the track - and are being outfitted with new $3 \mathrm{~mm}(85-105 \mathrm{GHz})$ and $12 \mathrm{~mm}(16-26 \mathrm{GHz})$ receiver systems. The sixth antenna is fixed at a station $3 \mathrm{~km}$ west of the track's end and will likely be outfitted with a $12 \mathrm{~mm}$ receiver only. At the time of the meeting, a 3-element prototype system was available at frequencies of $85-91 \mathrm{GHz}$ and $16-22 \mathrm{GHz}$; for the current status of the upgrade see the online documentation for observers (http://www.atnf.csiro.au/observers/).

Designed primarily as a $\mathrm{cm}$ array, the ATCA suffers from several problems as a mm array: a small primary beam, few baselines, poor aperture efficiency, and a far from ideal site at an elevation of $200 \mathrm{~m}$. In addition, the present correlator provides a maximum bandwidth of $128 \mathrm{MHz}$ at each of two frequencies, much less than the $1 \mathrm{GHz}$ or more that is typical at mm wavelengths (see Table 1). The velocity range that can be achieved by placing the two frequency windows side-by-side (allowing for some overlap) is limited to $\sim 600 \mathrm{~km} \mathrm{~s}^{-1}$ at $90 \mathrm{GHz}$. This 
Table 1. Upgraded ATCA compared with other $\mathrm{mm}$ arrays

\begin{tabular}{llrlrlllll}
\hline Array & $\begin{array}{l}\text { Altitude } \\
(\mathrm{km})\end{array}$ & $n$ & $\begin{array}{l}D \\
(\mathrm{~m})\end{array}$ & $\begin{array}{r}n D^{2} \\
\left(\mathrm{~m}^{2}\right)\end{array}$ & $\eta_{A}$ & $\begin{array}{l}\text { No. of } \\
\text { Polns }\end{array}$ & $\begin{array}{l}\text { Frequency range } \\
(\mathrm{GHz})\end{array}$ & $\begin{array}{l}\text { BW } \\
(\mathrm{GHz})\end{array}$ & $\begin{array}{l}b_{\max } \\
(\mathrm{km})\end{array}$ \\
\hline ATCA & 0.2 & 5 & 22 & 2420 & 0.4 & 2 & $16-26,85-105$ & 0.3 & 3.0 \\
BIMA & 1.0 & 10 & 6.1 & 370 & 0.7 & 1 & $70-116,210-270$ & 1.6 & 1.5 \\
IRAM & 2.6 & 6 & 15 & 1350 & 0.7 & 1 & $80-116,210-250$ & 1.0 & 0.4 \\
NMA & 1.3 & 6 & 10 & 600 & 0.6 & 1 & $85-116,213-237^{\mathrm{a}}$ & 1.0 & 0.4 \\
OVRO & 1.2 & 6 & 10.4 & 650 & 0.7 & 1 & $86-116,210-270$ & 4.0 & 0.4 \\
\hline
\end{tabular}

${ }^{\text {aalso }} 126-152 \mathrm{GHz}$.

Key: $n$ - number of antennas; $D$ - diameter of each antenna; $\eta_{A}$ - aperture efficiency of each antenna; $\mathrm{BW}$ - continuum bandwidth after combining both sidebands or IFs, if available; $b_{\max }-$ maximum baseline.

limitation will be overcome once a new wideband $(2 \mathrm{GHz})$ FX correlator is installed, as described during the meeting by W. Wilson. That project, to be undertaken in conjunction with development for the Square Kilometre Array (SKA), is due for completion around 2006.

Despite the ATCAs limitations as a mm array, once the initial stage of the upgrade (fitting complete receiver systems on five antennas) is complete in 2003 , it will possess several unique strengths:

- enormous collecting area, giving it an effective aperture $\left(\eta_{A} n D^{2}\right)$ comparable to that of the Plateau de Bure interferometer;

- ability to observe two polarisations simultaneously;

- wide-bandwidth mixers based on indium phosphide MMIC technology, which need only be cooled to about $20 \mathrm{~K}$, as opposed to $4 \mathrm{~K}$ for typical SIS junctions;

- a maximum baseline of $3 \mathrm{~km}$, allowing resolution as fine as 0.2 once radiometric phase correction is implemented.

As discussed at the meeting by $\mathbf{T}$. Wong, mm interferometry is much more challenging than cm interferometry, as a result of more stringent technical requirements (e.g. surface accuracy, pointing, and baseline determination) and the strongly variable effects of the atmosphere. In particular, fluctuations in the $\mathrm{H}_{2} \mathrm{O}$ vapour layer lead to fluctuations in atmospheric opacity and observed interferometric phase that must be monitored with frequent calibrations. These fluctuations tend to be more severe in the daytime and in summer, so winter nights are expected to offer the best observing conditions. As the fluctuations occur on a range of size scales, longer baselines will suffer from greater atmospheric phase noise under the same observing conditions. Long-baseline experiments also suffer from a lack of absolute flux calibrators, since the planets, which are the best primary flux calibrators, are likely to be resolved out.

The effects of atmospheric phase noise can be largely undone if the relative $\mathrm{H}_{2} \mathrm{O}$ vapour column above each antenna in the direction of the source is known. One way to determine the water vapour column is to accurately measure the strength of an $\mathrm{H}_{2} \mathrm{O}$ emission line, such as that at $22.2 \mathrm{GHz}$. This 'water vapour radiometry' technique was discussed by R. Sault and is currently being tested at the ATCA, with some encouraging results. The system

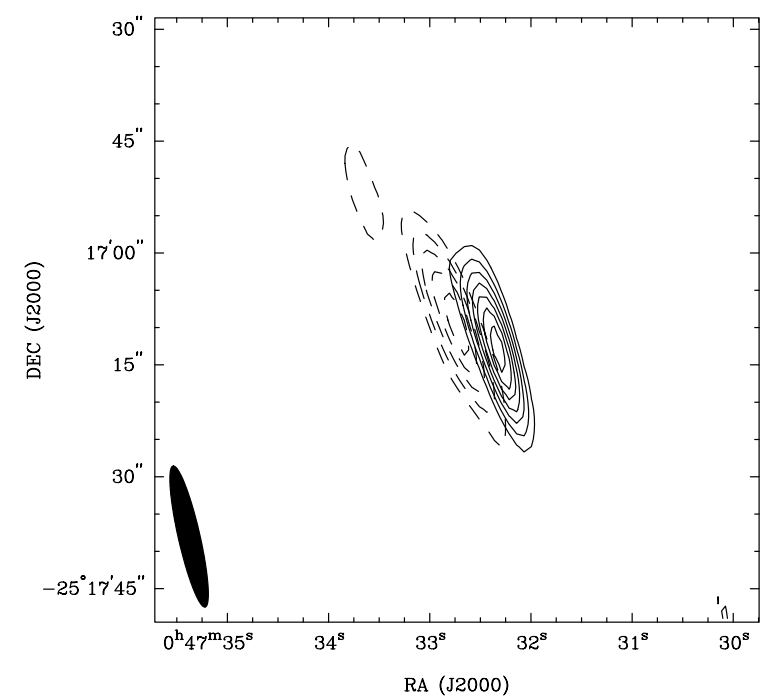

Figure 1 Deconvolved image of the nucleus of NGC 253 in the $\mathrm{HCO}^{+}(1-0)$ line. The beam size in 19 '. $^{\prime} \times 2$ 2'.9. Redshifted emision $\left(V_{\mathrm{LSR}}>230 \mathrm{~km} \mathrm{~s}^{-1}\right)$ is shown as solid contours, and blueshifted emission $\left(V_{\mathrm{LSR}}<230 \mathrm{~km} \mathrm{~s}^{-1}\right)$ as dashed contours. The data were taken with the 3-element system on 5 October 2001 over a period of $5.5 \mathrm{~h}$, with about $2 \mathrm{~h}$ of on-source integration.

observes the $22 \mathrm{GHz}$ line in four channels to better isolate line from continuum emission and reduce systematic errors.

B. Koribalski presented some of the first science observations made with the prototype $3 \mathrm{~mm}$ system, beginning with 'first light' in 2000 November. These included observations of molecular clouds in the Milky Way and LMC and detection of dense molecular gas in nearby galaxies such as NGC 253 (Figure 1) and Circinus. The highlight thus far has been the absorption spectrum of Centaurus $\mathrm{A}$ in the $\mathrm{HCO}^{+}$and $\mathrm{HNC}$ lines, which provides the first $\sim 1^{\prime \prime}$ resolution measurement of gas motions in front of the core of this radio galaxy (Figure 2). A search for CO absorption in the quasar PKS 1921-293 was also conducted.

R. Manchester presented the first image of the SN 1987A remnant at $17-19 \mathrm{GHz}$, made with the 3-element $12 \mathrm{~mm}$ system in 2001 October. The image, based on a 12 -h synthesis, shows a $\sim 20 \mathrm{mJy}$ source that is moderately resolved with a synthesised beam of 2 ". $8 \times 1$ 1. 8 (see Figure 3). A fit of a thin shell to the $u v$ data gives a diameter 


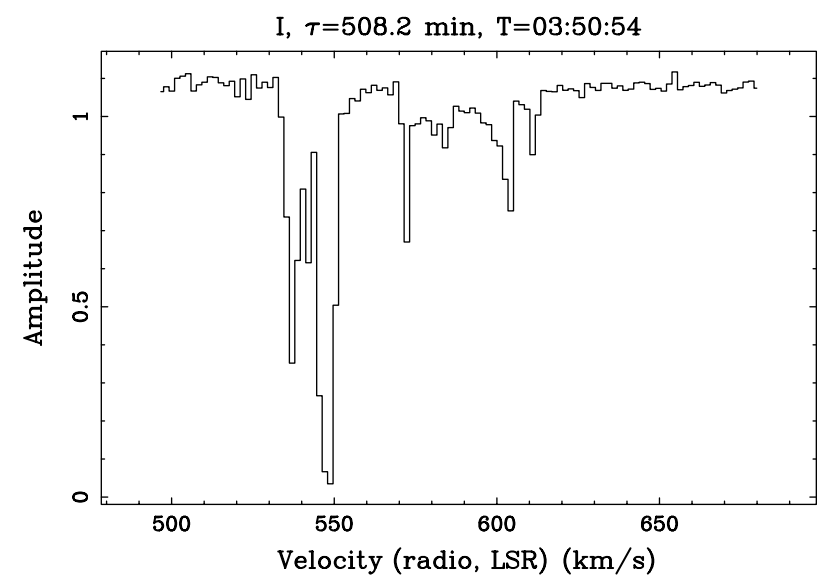

Figure 2 Absorption spectrum of the $\mathrm{HCO}^{+}(0-1)$ line in front of the continuum source in Centaurus A. Three hours of on-source integration were obtained with baselines of 46,77 , and $122 \mathrm{~m}$ on 6 October 2001. All three baselines have been averaged together to produce the final spectrum. The amplitude scale is in arbitrary units.

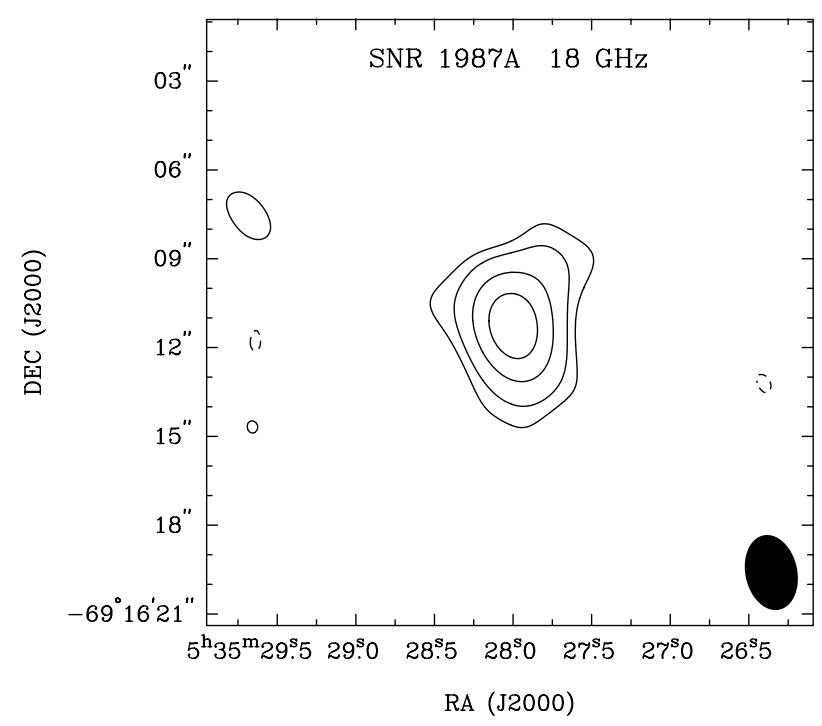

Figure 3 Image of the remnant of SN 1987A derived from a 10-h synthesis at the ATCA. Data from two frequencies $(17$ and $19 \mathrm{GHz})$ have been combined. The synthesised beam $\left(2{ }^{\prime \prime} 8 \times 1\right.$ 1" 8$)$ is shown at the lower right. The contour levels are $-1,1,2,5$, and $10 \mathrm{mJy}$ beam ${ }^{-1}$. Courtesy R. Manchester.

of 1 ..5 and a fit of a Gaussian gives a FWHM of 1". 1 . An IAU Circular (Manchester \& Gaensler 2001) has been released, and follow-up observations at higher resolution are planned.

\section{Molecular Clouds and Star Formation}

Studies of molecular clouds and star formation within them occupy a prominent place in mm astronomy, and were the subject of much discussion during the workshop. Indeed, as one of the ATCAs greatest attractions lies in its southern location, studies of Galactic star formation are poised to benefit immensely - most of the Galaxy's nearby star-forming regions (e.g. Chamaeleon, Corona Australis,
Lupus, Ophiuchus), not to mention the Galactic Centre, are located in the southern sky.

While almost all of the gas in molecular clouds consists of $\mathrm{H}_{2}$ and $\mathrm{He}$, these species do not emit radiation under conditions typically found in molecular clouds. Except in rare cases where $\mathrm{H}_{2}$ absorption against a UV source can be measured, the amount of molecular gas must be inferred indirectly. A common technique is to observe emission lines from tracer molecules like CO that have mm or submillimetre (sub-mm) rotational transitions. Not only can these lines provide information on the distribution and velocity of the molecular gas, but different molecular tracers can be used to probe different density regimes within a cloud.

The ATCAs ability to study nearby molecular clouds is limited by its small field of view, lack of sensitivity to extended structure, and inability to observe the bright $\mathrm{CO}(1-0)$ line at $115 \mathrm{GHz}$ (this last constraint may be overcome in a future upgrade). Thus, studies of the fragmentation of molecular clouds into clumps and cores (cf. Williams, Blitz, \& McKee 2000) are best undertaken with other instruments. Better suited to the ATCA will be studies of more distant clouds, such as in the Galactic Centre and Magellanic Clouds, and studies of dense molecular cores where star formation is occurring or likely to occur.

\subsection{Molecular Clouds near the Galactic Centre}

Cornelia C. Lang proposed several experiments that the ATCA can perform to increase our understanding of the Galactic Centre. Long-term monitoring of the mm flux from the radio source Sgr A*, thought to be associated with the central black hole, will help to determine whether the circumnuclear accretion disk is transitory or stable. Existing $\mathrm{NH}_{3}, \mathrm{HCN}$, and $\mathrm{HCO}^{+}$images of the circumnuclear disk, taken with the VLA and BIMA interferometers, show filamentary streamers of molecular gas falling onto the disk, providing direct evidence of inward gas transport (McGary, Coil, \& Ho 2001; Wright et al. 2001). However, they are inconclusive on the issue of disk stability.

Single-dish mapping of molecular gas in the vicinity of the Galactic Centre Radio Arc has revealed that it is physically related to the ionised gas in this region as well as to the radio non-thermal filaments (NTFs). Recently, interferometric studies made with OVRO reveal a complex geometrical arrangement and kinematic structure in the molecular gas (Lang, Goss, \& Morris 2002). The associations between the ionised and molecular gas suggest that the action of energetic winds from young stellar clusters (such as the Arches cluster) plays an important role in this region. The NTFs are thought to trace a largescale, poloidal field normal to the Galactic plane in the inner $250 \mathrm{pc}$ of the Galaxy. The interfaces between the NTFs and molecular clouds may be sites where twisting and reversal of magnetic field lines occurs, followed by magnetic reconnection which accelerates the electrons to relativistic speeds (e.g., Serabyn \& Morris 1994). With its superior view of the Galactic Centre, high spectral 
resolution, and dual polarisation capability, the ATCA can help elucidate these processes.

Millimetre images of the Galactic Centre can be compared with the recent Chandra Galactic Center Survey (Wang, Gotthelf, \& Lang 2002), which covers the central $300 \times 100$ pc with a resolution $\left(\sim 1^{\prime \prime}\right)$ that is well matched to the ATCA. Such multiwavelength studies will help to resolve puzzles like the origin of the diffuse $\mathrm{Fe}$ line emission at $6.4 \mathrm{keV}$ from the Galactic Centre molecular louds: is this fluorescent line excited by an energetic outburst from Sgr A* (Koyama et al. 1996), or from a more local source of X-rays responsible for driving the expanding molecular shells in the region (Martín-Pintado et al. 1999)?

\subsection{Shocked Molecular Gas}

The structure and chemical composition of a shock in the ISM are controlled by the shock speed $v_{s}$. In J-type, or 'fast', shocks, which occur for $v_{s} \gtrsim 40 \mathrm{~km} \mathrm{~s}^{-1}$, molecular gas is dissociated and ionised to a substantial degree immediately upstream and downstream from the shock, both by collisions and by UV radiation produced at the shock front. It is only far downstream from the shock, where the gas cools to $\approx 500 \mathrm{~K}$, that molecule reformation occurs. J-type shocks are inefficient emitters of infrared line radiation, but strong emitters of far-infrared continuum (because the UV flux is reprocessed by grains). On the other hand, in C-type, or 'slow', shocks, which occur for $v_{s} \lesssim 40 \mathrm{~km} \mathrm{~s}^{-1}$, the shock front is broadened by ambipolar diffusion, and molecules are not dissociated to the same extent. The energy radiated by such shocks appears mainly in infrared lines (atomic and ionic fine-structure lines and molecular rotation-vibration transitions). Since the molecules are not destroyed, they can reach much higher temperatures than in J-type shocks (Kaufman \& Neufeld 1996), driving endothermic reactions and hence interesting chemistry.

An important consequence of $\mathrm{C}$-shocks discussed by M. Wardle at the meeting is the efficient production of $\mathrm{H}_{2} \mathrm{O}$ from atomic oxygen $\left(\mathrm{O} \rightarrow \mathrm{OH} \rightarrow \mathrm{H}_{2} \mathrm{O}\right)$. This can give rise to intense water maser emission at $22 \mathrm{GHz}$ and at sub-mm wavelengths (Kaufman \& Neufeld 1996). Within supernova remnants (SNRs), a strong X-ray flux releases high-energy electrons which can excite the Lyman and Werner bands of $\mathrm{H}_{2}$. The resulting far-UV flux can dissociate some of the $\mathrm{H}_{2} \mathrm{O}$ back into $\mathrm{OH}$ (Wardle 1999), thereby accounting for the strong $\mathrm{OH}$ maser activity at $1720 \mathrm{MHz}$ associated with SNRs (Frail, Goss, \& Slysh 1994). The associations between shocked $\mathrm{H}_{2}$ and $\mathrm{OH}$ masers in the SNRs G359.1-0.5 (Lazendic et al. 2002) and G349.7+0.2 (J.S. Lazendic et al., in preparation) are consistent with this picture, but direct observations of $\mathrm{H}_{2} \mathrm{O}$, using the ATCA at $22 \mathrm{GHz}$, would be valuable.

Wardle also suggested a number of lines in the $3 \mathrm{~mm}$ band which could be used to probe shock regions. Good targets are the interaction regions between a supernova remnant and a cloud; many are distant, so a $30^{\prime \prime}$ field of view is acceptable. If $\mathrm{X}$-ray induced dissociation of water is indeed occurring, one would also expect the $\mathrm{SO}_{2} / \mathrm{SO}$ ratio to be affected, since $\mathrm{SO}_{2}$ is more easily dissociated than SO. This could be tested by observations of the $\mathrm{SO}\left(3_{2}-2_{1}\right)$ transition at $99.3 \mathrm{GHz}$ and the $\mathrm{SO}_{2}\left(3_{13}-2_{02}\right)$ transition at $104 \mathrm{GHz}$. With the existing prototype system, the ATCA can already image the shock-tracing $\mathrm{SiO}(2-1)$ line at $86 \mathrm{GHz}$. The presence of $\mathrm{SiO}$ in the gas phase is an indication of grain sputtering (Schilke et al. 1997).

\subsection{Identifying Molecular Cores}

As reviewed by T. Bourke, molecular cores have typical masses of $1-10 \mathrm{M}_{\odot}$ and sizes of $0.1-0.5 \mathrm{pc}$. They therefore occupy a small fraction of the mass and an even smaller fraction of the volume of an entire cloud. Since cores with embedded young stellar objects (YSOs) will be heated, they can be detected in wide-field maps of molecular clouds in infrared or sub-mm dust emission. Such maps will be provided by focal plane arrays on singledish telescopes, such as the SEST Imaging Bolometer Array (SIMBA), and by an upcoming Legacy project on the Space Infrared Telescope Facility (SIRTF). With its high resolution and sensitivity, the ATCA will be important for detailed studies of the cores identified by such surveys.

Starless cores, which by definition are not (yet) infrared sources, are probably best identified using line emission from dense gas tracers such as $\mathrm{NH}_{3}(23 \mathrm{GHz}), \mathrm{CS}$ (98 GHz), and $\mathrm{N}_{2} \mathrm{H}^{+}(93 \mathrm{GHz})$. The system of $\mathrm{NH}_{3}$ lines is also useful for determining densities and temperatures within cores. Temperature diagnostics are essential because observed linewidths may be dominated by turbulent motions.

While nearby clouds like Taurus are important laboratories for studying low-mass star formation, identifying sites where massive $\left(>10 \mathrm{M}_{\odot}\right)$ star formation is occurring is considerably more difficult, due to the rapid evolutionary timescales for massive stars, their tendency to form in clusters where source confusion is a problem, and their relative scarcity (hence, they tend to be observed at larger distances). The basic technique, as outlined by Bourke, is to search for cores with dense gas (as traced by CS emission) and hot dust (as traced by IRAS far-infrared fluxes) where the massive protostar has not yet ionised its surroundings (i.e., no detectable $5 \mathrm{GHz}$ emission from an HII region). A useful starting point would be the catalogue by Bronfman, Nyman, \& May (1986).

Masers are another important signpost for massive star formation, as pointed out by V. Minier. Class II methanol masers, especially the lines at 6.7 and $12.2 \mathrm{GHz}$, appear to be strongly correlated with the early stages of massive star formation, namely outflows and hot molecular cores, but maser activity apparently peaks before the emergence of an ultracompact HiI region (Minier, Conway, \& Booth 2001).

\subsection{From Dense Cores to Protostellar Disks}

The formation of protostellar disks is still poorly understood. Schematically, it appears that dense cores form within molecular clouds (perhaps at the intersections of 
filamentary structure) and then collapse gravitationally to form rotationally supported disks. Yet the dynamical processes and timescales involved in disk formation are still quite uncertain. A useful idealised model (neglecting rotation and magnetic fields) is the 'inside-out' collapse model of Shu (1977), which represents the collapse of a singular isothermal sphere. This model predicts powerlaw density and velocity profiles of $n(r) \propto r^{-1.5}$ and $v(r) \propto r^{-0.5}$ in the inner, collapsing regions (see Evans 1999).

M. Hunt described how mm spectroscopy can be used to determine chemical abundances and physical conditions in molecular cloud cores. She presented the results of single-dish studies of southern molecular clouds with Mopra and SEST. Of particular interest are molecules that can be observed at several optically thin transitions, so that a 'rotation diagram' (Goldsmith \& Langer 1999) can be used to infer the kinetic temperature, under the assumption that the level populations are in local thermodynamic equilibrium (LTE). Corrections for departures from LTE can be made using the large velocity gradient (LVG) approximation. Among the potential tracers for ATCA studies are $\mathrm{CH}_{3} \mathrm{OH}$ (at least 7 thermal transitions from $86-115 \mathrm{GHz}$ ), $\mathrm{HC}_{3} \mathrm{~N}$ (3 transitions), and OCS (3 transitions). The ATCA will be able to perform these studies at much higher resolution, reducing the uncertainties associated with averaging over a finite beam.

T. Bourke reviewed the rapidly growing literature concerned with spectroscopic infall signatures in dense cores. The most common signature, observed in optically thick lines like $\mathrm{HCO}^{+}$, is an asymmetric line profile skewed towards the blue, since the red side of the line is formed at larger (hence cooler) radii from the central source than the blue side (see explanation in Evans 1999). In addition, the presence of a cold, static envelope outside the collapse region leads to a self-absorption feature near the systemic velocity. Examples of line profiles that match these predictions are given in studies by Gregersen et al. (1997) of embedded YSOs and Lee, Myers, \& Tafalla (1999) of starless cores. These authors also show that blue-skewed profiles are not seen in optically thin tracers like $\mathrm{N}_{2} \mathrm{H}^{+}$or $\mathrm{H}^{13} \mathrm{CO}^{+}$, in agreement with infall models.

While most of these observations were made with single-dish telescopes, high-resolution data are important in that they allow better discrimination between the different effects (infall, rotation, outflow, and turbulence) which can be superposed in a single-dish beam. Indeed, the bestknown candidate for a collapsing protostar, in the isolated globule B335 (Zhou et al. 1993; Choi et al. 1995), looks less clear-cut when viewed at higher resolution (Wilner et al. 2000). This raises the question of whether single-dish data alone can sufficiently constrain the range of possible models. To date, the most convincing evidence for infall motions - line profiles with the 'inverse P Cygni' shape (blueshifted emission with redshifted absorption) - has been gathered with interferometers (e.g., Di Francesco et al. 2001), where the beam is sufficiently small to see the infalling gas in absorption.
G. Blake pointed out that high angular resolution is also needed to study the transition from the embedded phase, where most of the gas is in an infalling envelope, to the $\mathrm{T}$ Tauri phase, where most of the gas is in a rotating disk, as the angular size scales involved are $\sim 1000 \mathrm{AU}$, or $7^{\prime \prime}$ at the distance of Taurus. This transition appears to occur quite rapidly, given the apparent paucity of transitional forms (one example is proposed by Hogerheijde 2001). Disk envelopes can be traced via the $\mathrm{HCO}^{+}$and $\mathrm{HCN}$ lines, both of which are accessible with the ATCA prototype system, while the disk itself should be traceable in continuum emission, especially once the wideband correlator is available.

\subsection{Modelling Disk Evolution}

As reviewed by $\mathbf{S}$. Maddison at this meeting, simulations are an important tool for understanding the evolution of protostellar disks. Massive disks are susceptible to gravitational instabilities, which can induce mass accretion and hence feed back on the gravitational potential. These effects are difficult to model analytically but are wellsuited for N-body simulations. An important observational input is the mass surface density of the disk, which can be crudely estimated from the mass and radius. In the early stages of accretion, when the disk is massive, gravitational instabilities dominate and can lead to spiral arm formation or disk fragmentation - which can in turn lead to binary or giant planet formation. In later stages, most of the mass has accreted to the centre, the disk rotation is nearly Keplerian, and accretion is driven by turbulent viscosity.

A nice example of concordance between theory and observation occurs in the case of circumbinary disks. Subarcsecond-resolution observations of GG Tau (Guilloteau, Dutrey, \& Simon 1999) show a central hole in the dust distribution, consistent with the gap that should be cleared by resonant torques in a binary system. Observations at still higher resolution, however, will be needed to test the more detailed predictions of the simulations, such as the formation of bisymmetric spiral structure and accretion streams feeding the stars.

\subsection{Disks around pre-Main Sequence Stars}

G. Blake reviewed observations of protoplanetary disks, such as those associated with $\mathrm{T}$ Tauri stars. With radii of order $100 \mathrm{AU}$ ( $1^{\prime \prime}$ at the distance of Taurus), subarcsecond resolution will be needed to study such disks. Such resolution has recently been achieved by other $\mathrm{mm}$ arrays (e.g., Simon, Dutrey, \& Guilloteau 2001, using the IRAM Plateau de Bure), and should be achievable by the ATCA once the radiometric phase correction system is operational. Even with a small number of baselines and relatively incomplete coverage of the visibility plane, the basic structure of disks can be investigated by modelling the visibilities directly (e.g., Guilloteau \& Dutrey 1998).

Molecular lines can be a valuable probe of disk kinematics, but the large ranges in density and temperature around YSO accretion disks lead to a complex chemistry 
which is vital to understand. An important phenomenon is the depletion of molecules onto dust grains that occurs primarily at high densities and low temperatures but can be reversed by irradiation and shocks. The commonly employed molecular tracer $\mathrm{CO}$, for instance, may be depleted even when $\mathrm{H}_{2}$ remains in the gas phase. Such 'freeze-out' is especially likely near the disk midplane, where densities are highest. Where CO is detectable, it is likely to be optically thick and trace only the disk's outer layers; observing rarer $\mathrm{CO}$ isotopes, such as ${ }^{13} \mathrm{CO}$ $(110.2 \mathrm{GHz})$ and $\mathrm{C}^{18} \mathrm{O}(109.8 \mathrm{GHz})$, will be crucial for probing the vertical structure of disks. This will be a key focus for future high-resolution studies, and a strong argument for $110 \mathrm{GHz}$ capability at the ATCA.

Another way to trace the disk kinematics is via maser emission. The relative fringe phase between maser spots is insensitive to baseline errors and atmospheric effects (Wright \& Plambeck 1983), and can be used to deduce their relative positions to hundredths of an arcsecond if the spots are separated in velocity (e.g., Plambeck, Wright, \& Carlstrom 1990). In addition, once the instrumental baseline has been determined by observing quasars across a wide range of hour angles and declinations, absolute position information is also available (e.g., Forster, Welch, \& Wright 1978).

For deducing physical quantities such as density, temperature, and chemical composition, observations of multiple lines, including (for density and temperature) transitions between different energy levels of the same molecule, are necessary. Because of the ATCAs limited frequency range, many of the commonly used molecular tracers will only be observable in a single transition (generally the $J=1 \rightarrow 0$ line). Nonetheless, an interferometer map may still provide useful constraints on source geometry to complement single-dish measurements. Examples of studies that have exploited this method include Dutrey, Guilloteau, \& Guelin (1997) and Hogerheijde et al. (1997). Infrared spectra can also provide complementary information on physical conditions, with the advantage that many lines can be observed simultaneously in a given spectrum (see e.g. the review by van Dishoeck \& Blake 1998).

\subsection{Bipolar Outflows}

Outflows from star-forming cores are ubiquitous, as pointed out by T. Bourke, but are often so embedded in dust that they can only be studied in the radio. Fine velocity resolution is critical, as the outflow speeds can be as little as a few $\mathrm{km} \mathrm{s}^{-1}$. Spatial resolution is essential as well, especially in crowded regions where identifying the source of the outflow can be problematic. Some of the obvious southern targets are regions around $\rho$ Ophiuchi, $\mathrm{R}$ Coronae Australis, and Circinus.

While CO is the usual tracer because of its high abundance and low critical density, other molecular lines (e.g. from $\mathrm{CS}$ or $\mathrm{NH}_{3}$ ) can provide additional information. Of particular importance for the ATCA in the near term is the thermal $\mathrm{SiO}$ line at $86.8 \mathrm{GHz}$, which falls within one of the frequency windows available to the 3-element prototype system. The $\mathrm{SiO}$ molecules are liberated from dust grains shocked by the outflow, and thus trace the outflow more directly than a general gas tracer like CO. The presence of an outflow is often indicated in singledish spectra by broad line wings at relative velocities of up to $\sim 60 \mathrm{~km} \mathrm{~s}^{-1}$.

Although the origin of bipolar outflows remains controversial (see reviews by Shu et al. 2000; Königl \& Pudritz 2000), there is little doubt that magnetic fields play an important role in accelerating and collimating the observed jets. Magnetic fields in dense molecular regions are usually revealed by the linear polarisation of thermal emission from spinning dust grains. In regions where the continuum is weak, it is still possible to detect polarisation in molecular line transitions due to the Goldreich-Kylafis (Goldreich \& Kylafis 1981) effect, although this effect is rarely observed. For recent detections see Greaves et al. (1999) and Girart, Crutcher, \& Rao (1999). With its exceptional sensitivity and dual polarisation capability, the ATCA should be in a good position to exploit this technique.

R.G. Smith stressed the importance of complementary infrared observations. IR absorption spectra are sensitive to the presence of ices, since the same molecule produces different absorption profiles in the gas vs solid phase. Optical and near-infrared polarimetry can be used to reveal light that has been scattered by a disk. These techniques were illustrated in the case of $\mathrm{OH} 231.8+4.2$, an evolved star in which numerous molecular lines have been observed (Morris et al. 1987; Sánchez Contreras et al. 2000). Of particular interest among these is $\mathrm{HCO}^{+}$, which appears to be enhanced in the lobes of the outflow, perhaps due to shock-induced reactions.

\subsection{Debris Disks}

Late in their evolution, protostellar disks have dispersed most of their gas and a debris disk remains, with a mass only a few percent of the central star. Observations of such disks (and their immediate precursors, the disks around T Tauri and Herbig Ae/Be stars) were reviewed by Chris Wright. Debris disks were first identified as excess far-infrared emission around main sequence stars like Vega ( $\alpha$ Lyrae) and $\beta$ Pictoris. Ground-based (and more recently HST) imaging and spectroscopy revealed dusty disks around these stars, sometimes with warps and gaps indicative of planetary perturbers.

A primary motivation for studying these systems is to determine the timescale for disk dispersal, which is important for constraining theories of planet formation. This is best done by comparing the dust emission from disks in various stages of evolution (e.g., Holland et al. 1998). Although inferring dust masses from mm or sub$\mathrm{mm}$ continuum emission is hardly straightforward, one can get a handle on the grain properties using mid-infrared spectroscopy of the 10 and $20 \mu \mathrm{m}$ silicate features.

Studying the disk rotation and gas-phase chemistry requires molecular line observations. However, searches at $\mathrm{mm}$ wavelengths for gas in debris disks have generally 
been unsuccessful (e.g., Liseau \& Artymowicz 1998). Direct observations of $\mathrm{H}_{2}$ with the Infrared Space Observatory (ISO) indicate that there is indeed molecular gas present (Thi et al. 2001), so CO must be strongly depleted (by 2 to 3 orders of magnitude) compared to typical regions of molecular clouds. The reason for this depletion could be a combination of photodissociation and freeze-out onto grains. In addition, at temperatures above $20 \mathrm{~K}$, the $J=2$ and higher levels of $\mathrm{CO}$ are preferentially populated compared to $J=1$, and the $\mathrm{CO}$ (1-0) line is thus relatively weak (Liseau \& Artymowicz 1998). Indeed, the disk around the nearby T Tauri star TW Hya has been detected more strongly in the higher $J$ transitions (Kastner et al. 1997), with no CO(1-0) detected at Mopra (upper limit $\sim 0.3 \mathrm{~K}$ in a $30^{\prime \prime}$ beam). These results suggest that heating of the disk is rather efficient, probably due to flaring of the outer parts of the disk (cf. HST observations by Burrows et al. 1996).

\section{Interstellar and Circumstellar Masers}

\subsection{Methanol Masers}

Methanol $\left(\mathrm{CH}_{3} \mathrm{OH}\right)$ masers are generally found in active star-forming regions, and have traditionally been divided into two empirical classes. Class I masers are wellseparated from compact continuum sources and $\mathrm{OH}$ masers. Class II sources are generally associated with ultracompact HII (UCHII) regions, although this association has become less convincing with higher resolution data. Nonetheless, since certain maser lines are only seen in Class I sources and the rest only in Class II sources, the distinction between the two classes appears to have a physical basis. Methanol maser lines have been detected at numerous frequencies from 6.7 to $157 \mathrm{GHz}$, with the brightest lines being the Class II transitions at 6.7 and 12.2 GHz. The maser 'spots' themselves have sizes of order 1 milliarcsecond, although they are typically found in clusters of up to an arcsecond in size.

V. Minier reviewed observations of the spatial distribution of masers, many of them conducted at milliarcsecond resolution with VLBI. Interestingly, it appears that the 6.7 and $12.2 \mathrm{GHz}$ masers are often offset from their nearest UCHII regions by several arcseconds (Walsh et al. 1998; Minier et al. 2001). In these cases, they appear to coincide instead with hot molecular cores (HMCs) seen in $\mathrm{NH}_{3}$ and $\mathrm{CH}_{3} \mathrm{CN}$. A possible evolutionary scenario was presented, in which $\mathrm{CH}_{3} \mathrm{OH}$ masers form in the protostellar (HMC) phase, the young star begins to ionise its surroundings and destroy the masers, and finally an UCHII region emerges without maser activity. Testing this scenario requires resolving individual protostars using high-resolution instruments, inferring their masses from continuum fluxes, and inferring their ages by comparing observed spectral line ratios with chemical models (e.g., Rodgers \& Charnley 2001). Much of this work should be possible with the ATCA.

A. Sobolev discussed how physical conditions can be inferred from observations of molecular masers and thermal lines. Besides $\mathrm{CH}_{3} \mathrm{OH}$, a number of other maser transitions can be studied, including $\mathrm{H}_{2} \mathrm{O}, \mathrm{OH}, \mathrm{SiO}$, and $\mathrm{NH}_{3}$. Among the transitions of particular interest for the ATCA upgrade is the $95.2 \mathrm{GHz}$ Class $\mathrm{I} \mathrm{CH}_{3} \mathrm{OH}$ maser. A southern survey of these masers was recently conducted with the Mopra telescope by Val'tts et al. (2000). The spectra of $\mathrm{CH}_{3} \mathrm{OH}$ masers often show multiple, independently varying components that are generally attributed to motions within the molecular cloud, and can thus be used to probe the turbulent velocity spectrum. For example, the positions and spectra of masers near $25 \mathrm{GHz}$ detected in the Orion Molecular Cloud (Johnston et al. 1992) can be modelled in terms of Kolmogorov-like turbulence, although the spectrum appears to fall more steeply with wavenumber than the standard Kolmogorov law (Sobolev, Wallin, \& Watson 1998). Meanwhile, thermal lines, such as $\mathrm{CS}(2-1)$ at $96.4 \mathrm{GHz}, \mathrm{SiO}(2-1)$ at $86.8 \mathrm{GHz}$, and $\mathrm{CH}_{3} \mathrm{OH}\left(2_{K}-1_{K}\right)$ at $96.7 \mathrm{GHz}$, provide useful information about temperature, density, and shock chemistry, while high-resolution (interferometric) mapping can be used to study jets and shocks.

A more detailed look at the excitation of Class II $\mathrm{CH}_{3} \mathrm{OH}$ masers was provided by D. Cragg. Although the formation of inverted populations ('pumping') that can lead to masing remains an area of ongoing investigation, Class II transitions are thought to be pumped radiatively by infrared photons from the warm dust surrounding young massive stars. While the IR photons produce the population inversion, bright masers are the result of high $\mathrm{CH}_{3} \mathrm{OH}$ abundance, together with beaming and/or amplification of the UCHII background continuum. Sobolev \& Deguchi (1994) and Sobolev, Cragg, \& Godfrey (1997b) have modelled these processes in detail to reproduce the high observed brightness temperatures of the 6.7 and $12.2 \mathrm{GHz}$ masers. Strong masing is found to occur for dust temperatures $T_{d}>150 \mathrm{~K}$, gas densities $10^{6}<n_{\mathrm{H}}\left[\mathrm{cm}^{-3}\right]<10^{8}$, and $\mathrm{CH}_{3} \mathrm{OH}$ abundances relative to $\mathrm{H}_{2}$ of $>10^{-7}$. The models also predict Class II masers at 81.0, 85.6, 86.6, 86.9, 94.5, 108.9, and $111.2 \mathrm{GHz}$ (Sobolev, Cragg, \& Godfrey 1997a), in addition to the relatively strong line at $107.0 \mathrm{GHz}$, which has already been detected in over 25 sources (Val'tts et al. 1999; Caswell et al. 2000). Observational progress in detecting new maser transitions has been rapid, with recent detections of the 86.6 and $86.9 \mathrm{GHz}$ masers in the prototypical Class II source $\mathrm{W} 3(\mathrm{OH})$ reported by Sutton et al. (2001) using BIMA, and of the $85.6,86.6$, and $86.9 \mathrm{GHz}$ masers in the rich southern source G345.01+1.79 reported by Cragg et al. (2001) using SEST and Mopra.

\subsection{Late-Type Variable Stars}

Molecular masers are also observed around late-type variable stars such as Miras, which are characterised by pulsation periods in the range of $\sim 100$ to $\sim 500$ days, strong molecular bands in their optical spectra ( $\mathrm{TiO}$ and VO for type $\mathrm{M}, \mathrm{ZrO}$ for type $\mathrm{S}, \mathrm{CN}$ and $\mathrm{C}_{2}$ for type $\mathrm{C}$ ), and bright Balmer emission lines. The Balmer lines are produced by strong atmospheric shocks driven by 
the stellar pulsations; the outgoing, spherical pressure wave steepens to form a shock as it propagates into a decreasing density gradient. Mass loss at a rate of up to $10^{-5} \mathrm{M}_{\odot} \mathrm{yr}^{-1}$ creates a circumstellar envelope which hosts masers such as $\mathrm{OH}, \mathrm{H}_{2} \mathrm{O}, \mathrm{SiO}$, and $\mathrm{HCN}$. The $\mathrm{SiO}$ and $\mathrm{HCN}$ masers are believed to arise close to the stellar photosphere $\left(R \sim 10^{14} \mathrm{~cm}\right)$, whereas the $\mathrm{H}_{2} \mathrm{O}$ masers are associated with the expanding shell at distances $R \sim 10^{14}$ $10^{16} \mathrm{~cm}$, and $\mathrm{OH}$ masers with the outer parts of the shell at distances $R \gtrsim 10^{16} \mathrm{~cm}$.

The light curves of late-type variables vary with wavelength, as discussed by G. Rudnitskij. For example, the near-infrared maxima lag behind the optical maxima by about $0.1 P$, where $P$ is the period (Lockwood \& Wing 1971). The $\mathrm{H}_{2} \mathrm{O}$ maser flux also appears to lag behind the optical flux, by about 0.05-0.35 P in RS Virginis (Lekht et al. 2001). Such a large phase lag appears to rule out radiative pumping; instead, it is proposed that the arrival of a periodic shock into the masing region leads to enhanced collisional pumping of the maser (Rudnitskij \& Chuprikov 1990). Consistent with this hypothesis is the presence of a peak in the cross-correlation function at a lag of 3-5 $\mathrm{P}$ (where $P \approx 1 \mathrm{yr}$ ), roughly the time for a shock to travel from the stellar surface to the region where masers can form $\left(R \approx 10^{14} \mathrm{~cm}\right)$. Shock pumping is also suggested for the $\mathrm{H}_{2} \mathrm{O}$ maser flare in $\mathrm{R}$ Leo that was observed $\sim 18$ months after an $\mathrm{H} \alpha$ flare (Esipov et al. 1999). Although the $\mathrm{H}_{2} \mathrm{O}$ and $\mathrm{SiO}$ maser spots are expected to be unresolved by the ATCA, it was noted that if they are sufficiently bright and appear at distinct velocities, then their relative positions can be determined to a fraction of a beam (Plambeck et al. 1990; see Section 3.6).

Rudnitskij closed his presentation with the provocative suggestion that $\mathrm{H} \alpha$ flares may be due to a Jovian-sized planet in an eccentric orbit at a few AU, which has survived the red giant phase and excites strong atmospheric shocks during each periastron passage. If the stellar mass loss is asymmetric, a highly eccentric planetary orbit can be maintained against the circularising action of viscous torques.

\section{Interstellar Medium in Galaxies}

S. Curran noted that the ATCA is well suited to mapping the spatial distribution of molecular gas within Seyfert galaxies. Notably, the two nearest starburst/Seyfert hybrids, NGC 4945 and Circinus, are only observable with southern telescopes. An important observation would be to demonstrate the ability of a dusty circumnuclear torus to collimate a molecular outflow: Curran et al. (1999) argued for the existence of such an outflow in Circinus based on SEST mapping, but higher resolution observations are needed to confirm its association with the observed ionisation cone (Veilleux \& Bland-Hawthorn 1997, and references therein).

Another unresolved issue centres on the question of how well the dense gas tracer HCN correlates with star formation. NGC 4945 shows a higher $\mathrm{HCN} / \mathrm{CO}$ ratio, and a higher HCN/FIR (far-infrared) ratio, than Circinus despite lower gas densities $\left(\sim 10^{4}\right.$ vs $10^{5} \mathrm{~cm}^{-3}$ ) (Curran et al. 2001). In light of BIMA maps of the Seyfert galaxy NGC 1068 which show that most of the HCN is concentrated towards the centre, whereas $\mathrm{CO}$ and star formation are located in a ring $\sim 1 \mathrm{kpc}$ in radius (Helfer \& Blitz 1995), this suggests that much of the HCN emission in NGC 4945 comes from more diffuse gas associated with the dusty torus in the inner $\sim 10 \mathrm{pc}$, whereas in Circinus it is associated with star-forming cores in the $\sim 500 \mathrm{pc}$ radius molecular ring. However, better angular resolution will be needed to test this hypothesis.

M. Dopita discussed the implications of recent theoretical work on the predicted spectrum of dust emission at $\mathrm{mm}$ wavelengths. First of all, while the dust absorption coefficient $\kappa$ is usually assumed to scale as $\kappa \propto \nu^{0.7}$ at low frequencies, implying a steeply declining emission spectrum with wavelength, laboratory measurements by Mennella et al. (1998) indicate that $\kappa$ (and hence the emissivity) is strongly temperature-dependent, so that hot dust would show excess emission at long ( $\mathrm{mm}$ ) wavelengths. This could be an important consideration for interpreting $\mathrm{mm} / \mathrm{sub}-\mathrm{mm}$ continuum fluxes from galactic nuclei. Secondly, electric dipole radiation from small ( $\sim 100$ atoms) aspherical, rotating grains is predicted to peak at a rest frequency near $30 \mathrm{GHz}$ (Draine \& Lazarian 1998). This prediction follows from a consideration of several effects including 'plasma drag' (the interaction of the grain's dipole moment with passing ions) and 'electrostatic focusing' (the change in the collisional cross section due to electrostatic attraction or repulsion), and may explain the excess background emission near this frequency observed by Leitch et al. (1997) and others. Finally, magnetic dipole radiation can be emitted by dust containing ferromagnetic materials (Draine \& Lazarian 1999), with emission peaking in the tens of $\mathrm{GHz}$ (depending on the characteristic gyrofrequency).

\section{High-Redshift Molecular Gas}

T. Wiklind reviewed the observability of molecular gas at high-redshift. Studies of emission and absorption are complementary, with emission lines providing global properties (gas content and rotational velocity) whereas absorption lines probe the gas on much smaller scales and with much greater sensitivity. Although the number of detections to date is small, the fact that the CO line has already been observed out to $z \sim 5$ has allayed concerns that low metallicities would render it undetectable. On the other hand, there is little doubt that metallicity does affect the $L_{\mathrm{CO}} / M_{\mathrm{H}_{2}}$ ratio (e.g., Maloney \& Black 1988), so the objects detected (especially in emission) are likely to be unusually luminous and dusty. Moreover, optimistic early assessments of the detectability of CO at $z>5$ (Silk \& Spaans 1997) have been questioned by more recent simulations (Combes, Maoli, \& Omont 1999).

As of 2001 November, thirteen CO emission sources had been detected at $z>1$ (all with interferometers, which 
$85-115 \mathrm{GHz}$

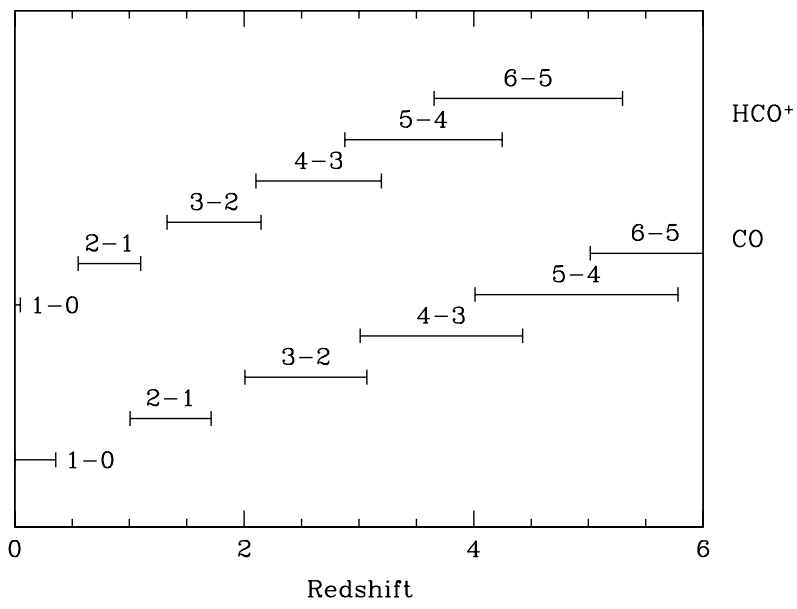

Figure 4 Observability of the $\mathrm{CO}$ and $\mathrm{HCO}^{+}$lines at high-redshift with a frequency coverage from $85-115 \mathrm{GHz}$. For each transition there is a redshift range (given by a horizontal bar) where the line falls within the observable band. Adapted from a figure by T. Wiklind.

provide the necessary gain stability for long integrations); more than half of the detected sources appear to be gravitationally lensed. The highest redshift source is at $z=4.69$ (Omont et al. 1996). Note that because of the increased cosmic microwave background (CMB) temperature at high-redshift, higher $J$ transitions of $\mathrm{CO}$ can be as bright or even brighter than the $J=1 \rightarrow 0$ transition. Thus, high-redshift sources can still be detected in the $3 \mathrm{~mm}$ band, as high $J$ transitions are redshifted into this band. Figure 4 shows the redshift coverage of the ATCA system, assuming its frequency range can be extended to $115 \mathrm{GHz}$.

Absorption lines can be detected out to any redshift as long as a suitably bright continuum source is available. For instance, one can achieve comparable signal-to-noise on the $z>0.89$ quasar PKS 1830-211 (Wiklind \& Combes 1996) as on a nearby galaxy like Cen A. The integrated opacity can be approximated as

$$
\int \tau_{v} d v \propto \frac{N_{\text {tot }}}{T_{x}^{2}} \mu_{0}^{2}
$$

where $N_{\text {tot }}$ is the total column density of the molecule, $T_{x}$ is the excitation temperature given by the level populations, and $\mu_{0}$ is the permanent dipole moment. This expression shows that absorption is particularly sensitive to cold gas, and that a molecule with a high dipole moment like $\mathrm{HCO}^{+}$can be as strong as $\mathrm{CO}$ in absorption, despite lower abundance. Including the $\mathrm{HCO}^{+}$series of lines allows for even more complete redshift coverage in the $3 \mathrm{~mm}$ band (Figure 4).

With its excellent sensitivity, the ATCA should be a superb tool for detecting molecules in high-redshift galaxies. Potential emission targets are best identified at sub-mm wavelengths (e.g., Frayer et al. 1998), where the strongly increasing dust emission with frequency offsets the dimming due to increased cosmological distance (Hughes 1996). The SCUBA and SIMBA instruments on the James Clerk Maxwell Telecope (JCMT) and the SEST respectively are being utilised for such searches. Redshifts can be determined by several techniques, listed here in order of decreasing accuracy: (1) optical spectroscopy (e.g., Ivison et al. 1998), (2) multi-colour optical photometry (e.g., Mobasher et al. 1996), or (3) the radio-to-sub-mm spectral index (Carilli \& Yun 1999). While optical redshifts (methods 1 and 2) are more accurate, Smail et al. (2000) have concluded that most of the SCUBA-detected sources are at $z \gtrsim 2$ and do not have optical identifications. The third method, based on the assumption that the observed correlation between radio and far-infrared emission in star-forming galaxies (Condon 1992) is valid at high-redshift, requires only flux measurements at 1.4 and $350 \mathrm{GHz}$.

Potential absorption targets can be selected from catalogues of radio-loud (especially mm-loud) QSOs. In principle, optical and/or near-infrared colours can be used to select quasars which are reddened by dust (Webster et al. 1995), as would be expected if there were intervening molecular gas. On the other hand, as noted by R. Webster and M. Drinkwater at the meeting, synchrotron emission may account for the reddened spectra in a large fraction of cases (Francis et al. 2001), highlighting the need for more refined selection criteria. Yet another problem is choosing the observing frequency in the absence of a reliable optical redshift (for either the background source or the absorber). Lacking redshift information, it should still be possible to scan across the $3 \mathrm{~mm}$ band in a reasonable amount of time (e.g., Wiklind \& Combes 1996), particularly once the ATCAs bandwidth has been upgraded.

An increased sample of molecular gas detections at high-redshift would benefit a variety of studies. By providing accurate redshifts for sub-mm sources, they would significantly improve our knowledge of the evolution of massive galaxies and of the star formation rate. In addition, by observing multiple transitions of the same molecule and deriving the excitation temperature, one can place upper limits on the CMB temperature as a function of redshift.

As discussed by M. Murphy and M. Drinkwater in the final talk of the workshop, high signal-to-noise $\mathrm{CO}$ and Hi spectra of absorption-line systems can be used to provide constraints on variations in the fine-structure constant ( $\alpha=e^{2} / \hbar c$ ) with redshift. Specifically, the ratio of the $\mathrm{HI}$ hyperfine to the $\mathrm{CO}$ rotational line frequency is proportional to $y=\alpha^{2} g_{p}$, where $g_{p}$ is the proton $g$-factor (Murphy et al. 2001a). A recent analysis by (Murphy et al. (2001a) leads to an upper limit of $\Delta \alpha / \alpha<3 \times 10^{-6}$ at redshifts of 0.25 and 0.68 , assuming a constant $g_{p}$. However, higher redshift systems are needed to test claims, based on optical QSO spectra, that $\Delta \alpha / \alpha$ deviates significantly from 0 at $z>1$ (Webb et al. 1999; Murphy et al. 2001b).

\section{Acknowledgments}

We would like to thank the staff and students of Melbourne University for helping to make the workshop a success. 
We also appreciate the generosity of the MNRF International Collaboration Committee, which helped to support the travel costs of many of our visitors. The Australia Telescope Compact Array is part of the Australia Telescope, which is funded by the Commonwealth of Australia for operation as a National Facility managed by CSIRO.

\section{References}

Bronfman, L., Nyman, L.-A., \& May, J. 1986, A\&AS, 115, 81

Burrows, C. J., et al. 1996, ApJ, 473, 437

Carilli, C. L., \& Yun, M. S. 1999, ApJ, 513, L13

Caswell, J. L., Yi, J., Booth, R. S., \& Cragg, D. M. 2000, MNRAS, 313, 599

Choi, M., Evans, N. J., Gregersen, E. M., \& Wang, Y. 1995, ApJ, 448, 742

Combes, F., Maoli, R., \& Omont, A. 1999, A\&A, 345, 369

Condon, J. J. 1992, ARA\&A, 30, 575

Cragg, D. M., Sobolev, A. M., Ellingsen, S. P., Caswell, J. L., Godfrey, P. D., Salii, S. V., \& Dodson, R. G. 2001, MNRAS, 323, 939

Curran, S. J., Johansson, L. E. B., Bergman, P., Heikkilä, A., \& Aalto, S. 2001, A\&A, 367, 457

Curran, S. J., Rydbeck, G., Johansson, L. E. B., \& Booth, R. S. 1999 , A\&A, 344, 767

Di Francesco, J., Myers, P. C., Wilner, D. J., Ohashi, N., \& Mardones, D. 2001, ApJ, 562, 770

Draine, B. T., \& Lazarian, A. 1998, ApJ, 494, L19

Draine, B. T., \& Lazarian, A. 1999, ApJ, 512, 740

Dutrey, A., Guilloteau, S., \& Guelin, M. 1997, A\&A, 317, L55

Esipov, V. F., Pashchenko, M. I., Rudnitskii, G. M., \& Fomin, S. V. 1999, AstL, 25, 672

Evans, N. J. 1999, ARA\&A, 37, 311

Forster, J. R., Welch, W. J., \& Wright, M. C. H. 1978, ApJ, 221, 137

Frail, D. A., Goss, W. M., \& Slysh, V. I. 1994, ApJ, 424, L111

Francis, P. J., Drake, C. L., Whiting, M. T., Drinkwater, M. J., \& Webster, R. L. 2001, PASA, 18, 221

Frayer, D. T., Ivison, R. J., Scoville, N. Z., Yun, M., Evans, A. S., Smail, I., Blain, A. W., \& Kneib, J.-P. 1998, ApJ, 506, L7

Girart, J. M., Crutcher, R. M., \& Rao, R. 1999, ApJ, 525, L109

Goldreich, P., \& Kylafis, N. D. 1981, ApJ, 243, L75

Goldsmith, P. F., \& Langer, W. D. 1999, ApJ, 517, 209

Greaves, J. S., Holland, W. S., Friberg, P., \& Dent, W. R. F. 1999 , ApJ, 512, L139

Gregersen, E. M., Evans, N. J., Zhou, S., \& Choi, M. 1997, ApJ, 484,256

Guilloteau, S., \& Dutrey, A. 1998, A\&A, 339, 467

Guilloteau, S., Dutrey, A., \& Simon, M. 1999, A\&A, 348, 570

Helfer, T. T., \& Blitz, L. 1995, ApJ, 450, 90

Hogerheijde, M. R. 2001, ApJ, 553, 618

Hogerheijde, M. R., van Dishoeck, E. F., Blake, G. A., \& van Langevelde, H. J. 1997, ApJ, 489, 293

Holland, W. S., et al. 1998, Nature, 392, 788

Hughes, D. H. 1996, in Cold Gas at High Redshift, ed. M. N. Bremer, P. P. van der Werf, H. J. A. Röttgering, \& C. L. Carilli (Dordrecht: Kluwer), 311

Ivison, R. J., Smail, I., Le Borgne, J.-F., Blain, A. W., Kneib, J.-P., Bezecourt, J., Kerr, T. H., \& Davies, J. K. 1998, MNRAS, 298, 583

Johnston, K. J., Gaume, R., Stolovy, S., Wilson, T. L., Walmsley, C. M., \& Menten, K. M. 1992, ApJ, 385, 232

Kastner, J. H., Zuckerman, B., Weintraub, D. A., \& Forveille, T. 1997, Science, 277, 67

Kaufman, M. J., \& Neufeld, D. A. 1996, ApJ, 456, 250

Königl, A., \& Pudritz, R. E. 2000, in Protostars and Planets IV, ed. V. Mannings, A. P. Boss, \& S. S. Russell (Tucson: University of Arizona Press), 759
Koyama, K., Maeda, Y., Sonobe, T., Takeshima, T., Tanaka, Y., \& Yamauchi, S. 1996, PASJ, 48, 249

Lang, C. C., Goss, W. M., \& Morris, M. 2002, AJ, submitted

Lang, C. C., Goss, W. M., \& Rodríguez, L. F. 2001, ApJ, 551, L143

Lazendic, J. S., Wardle, M., Burton, M. G., Yusef-Zadeh, F., Whiteoak, J. B., Green, A. J., \& Ashley, M. C. B. 2002, MNRAS, 331,537

Lee, C. W., Myers, P. C., \& Tafalla, M. 1999, ApJ, 526, 788

Leitch, E. M., Readhead, A. C. S., Pearson, T. J., \& Myers, S. T. 1997, ApJ, 486, L23

Lekht, E. E., Mendoza-Torres, J. E., Rudnitskij, G. M., \& Tolmachev, A. M. 2001, A\&A, 376, 928

Liseau, R., \& Artymowicz, P. 1998, A\&A, 334, 935

Lockwood, G. W., \& Wing, R. F. 1971, ApJ, 169, 63

Maloney, P., \& Black, J. H. 1988, ApJ, 325, 389

Manchester, R. N., \& Gaensler, B. M. 2001, IAUC, 7757, 2

Martín-Pintado, J., Gaume, R. A., Rodríguez-Fernández, N., de Vicente, P., \& Wilson, T. L. 1999, ApJ, 519, 667

McGary, R. S., Coil, A. L., \& Ho, P. T. P. 2001, ApJ, 559, 326

Mennella, V., Brucato, J. R., Colangeli, L., Palumbo, P., Rotundi, A., \& Bussoletti, E. 1998, ApJ, 496, 1058

Minier, V., Conway, J. E., \& Booth, R. S. 2001, A\&A, 369, 278

Mobasher, B., Rowan-Robinson, M., Georgakakis, A., \& Eaton, N. 1996, MNRAS, 282, L7

Morris, M., Guilloteau, S., Lucas, R., \& Omont, A. 1987, ApJ, 321,888

Murphy, M. T., Webb, J. K., Flambaum, V. V., Drinkwater, M. J., Combes, F., \& Wiklind, T. 2001a, MNRAS, 327, 1244

Murphy, M. T., Webb, J. K., Flambaum, V. V., Dzuba, V. A., Churchill, C. W., Prochaska, J. X., Barrow, J. D., \& Wolfe, A. M. 2001b, MNRAS, 327, 1208

Omont, A., Petitjean, P., Guilloteau, S., McMahon, R. G., Solomon, P. M., \& Pecontal, E. 1996, Nature, 382, 428

Plambeck, R. L., Wright, M. C. H., \& Carlstrom, J. E. 1990, ApJ, 348, L65

Rodgers, S. D., \& Charnley, S. B. 2001, ApJ, 546, 324

Rudnitskij, G. M., \& Chuprikov, A. A. 1990, AZh, 67, 293

Sánchez Contreras, C., Bujarrabal, V., Neri, R., \& Alcolea, J. 2000, A\&A, 357, 651

Schilke, P., Walmsley, C. M., Pineau des Forêts, G., \& Flower, D. R. 1997, A\&A, 321, 293

Serabyn, E., \& Morris, M. 1994, ApJ, 424, L91

Shu, F. H. 1977, ApJ, 214, 488

Shu, F. H., Najita, J. R., Shang, H., \& Li, Z.-Y. 2000, in Protostars and Planets IV, ed. V.Mannings, A. P. Boss, \& S. S. Russell (Tucson: University of Arizona Press), 789

Silk, J., \& Spaans, M. 1997, ApJ, 488, L79

Simon, M., Dutrey, A., \& Guilloteau, S. 2001, ApJ, 545, 1034

Smail, I., Ivison, R. J., Owen, F. N., Blain, A. W., \& Kneib, J.-P. 2000, ApJ, 528, 612

Sobolev, A. M., Cragg, D. M., \& Godfrey, P. D. 1997a, MNRAS, 288, L39

Sobolev, A. M., Cragg, D. M., \& Godfrey, P. D. 1997b, A\&A, 324, 211

Sobolev, A. M., \& Deguchi, S. 1994, A\&A, 291, 569

Sobolev, A. M., Wallin, B. K., \& Watson, W. D. 1998, ApJ, 498, 763

Sutton, E. C., Sobolev, A. M., Ellingsen, S. P., Cragg, D. M., Mehringer, D. M., Ostrovskii, A. B., \& Godfrey, P. D. 2001, ApJ, 554, 173

Thi, W. F., et al., 2001, Nature, 409, 60

Val'tts, I. E., Ellingsen, S. P., Slysh, V. I., Kalenskii, S. V., Otrupcek, R., \& Larionov, G. M. 2000, MNRAS, 317, 315

Val'tts, I. E., Ellingsen, S. P., Slysh, V. I., Kalenskii, S. V., Otrupcek, R., \& Voronkov, M. A. 1999, MNRAS, 310, 1077

van Dishoeck, E. F., \& Blake, G. A. 1998, ARA\&A, 36, 317

Veilleux, S., \& Bland-Hawthorn, J. 1997, ApJ, 479, L105

Walsh, A. J., Burton, M. G., Hyland, A. R., \& Robinson, G. 1998, MNRAS, 301, 640

Wang, Q. D., Gotthelf, E. V., \& Lang, C. C. 2002, Nature, 415, 148 
Wardle, M. 1999, ApJ, 525, L101

Webb, J. K., Flambaum, V. V., Churchill, C. W., Drinkwater, M. J., \& Barrow, J. D. 1999, Physical Review Letters, 82, 884

Webster, R. L., Francis, P. J., Peterson, B. A., Drinkwater, M. J., \& Masci, F. J. 1995, Nature, 375, 469

Wiklind, T., \& Combes, F. 1996, Nature, 379, 139

Williams, J. P., Blitz, L., \& McKee, C. F. 2000, in Protostars and Planets IV, ed. V. Mannings, A. P. Boss, \& S. S. Russell (Tucson: University of Arizona Press), 97
Wilner, D. J., Myers, P. C., Mardones, D., \& Tafalla, M. 2000, ApJ, 544, L69

Wright, M. C. H., Coil, A. L., McGary, R. S., Ho, P. T. P., \& Harris, A. I. 2001, ApJ, 551, 254

Wright, M. C. H., \& Plambeck, R. L. 1983, ApJ, 267, L115

Zhou, S., Evans, N. J., Koempe, C., \& Walmsley, C. M. 1993, ApJ, 404, 232 